\title{
Influence of Social Media Use on Face-to-Face Interaction Among University of Lagos Undergraduates
}

\author{
Akoja, Mofoluke I. Odozi, Amaka \\ Mass Communication Department, Veronica Adeleke School of Social Sciences, Babcock University
}

\begin{abstract}
The use of social media has brought with it tremendous benefits and at the same time negative influences have been observed especially on human interactions. Today, many youths appear to be stuck with different social media platforms in order to meet varying needs depending on the user and the features of such platforms. The result is a depleted quality and importance of face-to-face interaction which remains an important aspect of human interactions. This study investigates the influence of social media use on face-to-face interactions among University of Lagos undergraduates. Anchored on three theoretical assumptions namely uses and gratification, media dependency theory and media richness theories, the study adopted the mixed method research methods involving both qualitative and quantitative data gathered using two different instruments - questionnaire and focus group guide. Data were derived from 397 students from two departments. Findings showed that majority of the respondents are dependent on social media as they make use of its different platforms regularly for communication (80.3\%), with the most commonly used being Facebook (31\%). Whereas, face-to-face interaction is still a relevant form of communication because majority of the respondents agreed that it allows them to relate better (64.1) and is the most preferred (75.7\%). The study recommends enlightening students about the advantages and disadvantages of social media use especially as it concerns face-to-face interactions
\end{abstract}

Keywords: Influence, Social media, Face-to-face interaction

DOI: $10.7176 / \mathrm{NMMC} / 88-04$

Publication date: February $29^{\text {th }} 2020$

\section{Introduction}

Communication remains a global experience which affects the lives of every human being just as much as breathing is important and recognised as essential for humans. Globalisation is a major factor influencing how communication has evolved thereby taking on new and better forms. The introduction of web 2.0 technologies not only improved the performance of the internet but brought along interactive features which give users more innovative options when it comes to social interactions. Social media has become an essential and viable platform that has virtually connected the world, thus giving people both far and near the opportunity to interact and collaborate with one another thereby building stronger relationships. The different social media sites allow people to network, engage in online business transactions, buy goods and participate in political campaigns. Hence, this modern platform of social interaction has introduced significant, pervasive and profound changes to the communication process between organisations, communities and individuals (Zuo, 2015). Nonetheless, the use of social media has both positive and negative effects one of which is its influence on face to face interaction which hitherto was an unavoidable part of interpersonal communication.

Interpersonal communication can be referred to as the exchange of information among individuals and groups either using computer mediated communication tools like social media or face-to-face. It is an indispensable mode of communication which has to do with how people express their feelings and share information with one another using either verbal or non-verbal messages. The importance of relationship to human survival and growth was underscored by an English poet, Donne (1624), who says that no one is an island. Generally, face-to-face interaction remains an essential part of human interactions even with the advancement brought about by social media, relationships that were originally built and dependent on purely physical interaction has been improved giving rise to new patterns of interpersonal relationship.

Although, social media technologies first gained prominence in the early 1990s, the first modern social network called "SixDegrees.com" was established in 1997. This social media platform enabled people to set up their personal profiles and expand their circle of friends. Presently, there are popular sites like Facebook, Twitter that provide diverse interactive features for users. Initially, these platforms could only be accessed on computers, however, as a result of to the continuous technological advancements and increased popularity of smartphones, social media platforms began to modify to functioning through the use of web based applications that can run efficiently on smartphones providing more convenience to users. On the other hand, the increasing use of social media raises a lot of concern on the quality of interpersonal interactions people can experience with one another. It is a common site to walk into a place and find many people with their heads down busy with their cell-phones. What emerged as hobby for some computer literate has grown to be a way of life and a social norm (Boyd, 2007). This has raised a lot of concern on the changing nature of human interactions from face-to-face interactions to mediated forms especially through the social media. 
Incidentally, teenagers and youths are the victims of this new pattern as they are often more keen about embracing new technologies and becoming familiarised with them very quickly than adults. According to Godson and Chukwuemeka (2014), social media use among young people in Nigeria is starting to occupy other modes of communication particularly face-to-face interaction. Social media usage has gathered a lot of attention in a number of research studies except how it influences face-to-face interaction. In view of its growing importance, this study examines how the usage of social media has influenced face-to-face communication among youths.

\section{Statement of the Problem}

Social media is a virtual platform where people can interact with one another and build relationships irrespective of distance, place and time. Even though, people use these platforms to exchange information and interact, there is an increased dependence on these platforms and this has raised concerns on the less use of face-to-face interactions which characterised how people have always interacted. It is true that social media provides an easy and effective way for human interactions, however, it has been found to be the cause of reduced learning and research capabilities among youths. Although social media provides awareness on recent happenings in the world, nonetheless, research has shown that $29 \%$ of untrustworthy sexual conduct were initiated on social networking site (Guardchild, 2018). Previous studies have also revealed that overdependence on social media platforms like Facebook and WhatsApp by youths and teenagers has a negative impact on their interpersonal skills as well as their relationships which is now limited to online chatting, video calling, text messaging, among others (Ndunge, 2017). People often look out for hints, signs and cues about what type of behaviour is suitable and should be exhibited in the context and how well to interpret what others mean when communicating. Social media lacks the ability to offer these symbols and without these, people might find it hard to understand and decipher what the other person is truly saying. In the cooperate world, employers have found ways to beat the limitations of face-to-face communication warranted by such occasions such as job interviews and officials meetings by conducting such through video conferences on applications such as Skype. In as much as social media has tremendously improved human experience, it is slowly affecting the traditional face-to-face interaction in various ways especially in interpersonal relationships. There is no doubt that that social media reduces the quality of human communication as not all aspects of human expressions, for instance, non-verbal cues, can be effectively incorporated into this process. As such, exchange of meaning may be incomplete making communication haphazard and ineffective. Despite this, there has been a drastic change in the way people communicate such that they prefer communicating over social media than face-to-face interaction. According to Keller (2013), "we would preferably e-mail than meet or text than talk on the phone". The study therefore looked at the influence of social media on face-to-face interactions among youths and the factors contributing to this trend.

\section{Objective of the Study}

The study evaluated the influence of social media use on face-to-face interaction among University of Lagos undergraduates by specifically

1. identifying the pattern of social media usage among Unilag undergraduates.

2. determining the nature of face-to-face interaction among Unilag undergraduates.

3. investigating the perceived difference between online and face-to-face interactions by Unilag undergraduates.

4. examining the factors influencing social media interactions and face-to-face interactions among Unilag undergraduates.

\section{Theoretical Justification}

The study adopts the uses and gratification theory and the media dependency theory. This theory was postulated by Elihu Katz, Jay Blumler and Michael Gurevitch in 1974. As media users are increasingly confronted with different choices, this approach focuses on the audience who are the consumers of media and how they make use of the media for their personal benefits. The introduction of modern technology has changed our lives, the way we interact, communicate and operate. These uses and gratifications are established by the needs of media users which include information, entertainment, and education. McQuail (2010) states that media habits are centred on similarities of individual needs, interests and taste. People often make use of media contents for information, relaxation, companionship, diversion or 'escape'. The theory explains that media consumers are not circumstantially forced to use media without some element of preference. Before settling on a specific choice of media, users have their own reasoning and logic that guide their own preference. Users get to select the best media that best meets their communication needs or wants. If you look at it through a keen eye, you may realize that media sources are in competition to find users (Katz, et al 1974). Social media platforms come with different interesting features and they compete with one another in order to attract users. For instance, over time, 
Facebook tries to create similar outlooks to what a user may find on Instagram, in order to draw more users to the platform. In relations to this study, people have various reasons why they make use of certain media which could be learning or communicating because each medium is unique in its purpose (Spring, 2002). Although, the theory originated before the technological advances and the advent of social media, presently, the theory has more relevance than ever as a means of understanding how and why people make use of existing technologies such as mobile phones, social media, internet, video conferencing, etc. Uses and Gratification theory research in relations to social media usage explains that people seek for benefits such as interaction, information, and entertainment, among others from these platforms. For example, a need to express themselves, reinvent, identify with others and satisfy their cognitive needs.

An expansion of the uses and gratifications theory is the media dependency theory. Media dependency posits that that audience goals is the origin of dependency while uses and gratification emphasizes audience needs. Basically, the relationship between both theories is that there is an agreement that media use yields gratification. The media dependency theory also positions the audience as an active part of the communication process. Individuals have different goals and needs which could range from politics, to sports or entertainment which they would want to fulfil. Hence, the media is the key to finding the solution. Basically, the more a person relies on a medium to satisfy these needs, the more significant the medium turns out to be in the person's life and therefore the more effects the medium will have on the person (Ball-Rokeach \& Defleur, 1998). Whenever people need to satisfy their needs or goals, they resort to a certain media. According to Ball-Rokeach \& Defleur (1976) cited in Loges \& Ball-Rokeach (1993), this theory also explains that the amount of time expended using a medium describes the audience's dependency on the medium. It also states that a number of goals can be satisfied by one medium. Developing dependency on media depends on media availability, media capability and the relationship between the society and the audience. The study affirms the assumption of this theory by arguing that youths are becoming increasingly dependent on the social media especially because of the opportunity it gives to them to imitate other people's behaviours and lifestyles without them being aware and also the fact that it enables the users to evaluate their social environment. Another reason why social media may be becoming more dependable is the fact that it is more readily available, capable of meeting the different needs of the users as well as connecting user to the world. Users are able to interact just the way people interact face to face except this is done via technology.

\section{The role of interpersonal communication in human communication}

Interpersonal communication refers to an interactive exchange of information between two or more individuals. It takes cognisance of verbal and non-verbal cues and how they are used to achieve personal and social goals. According to Knapp (2002), interpersonal communication is regarded as "a mutual, continual process of sending, receiving, and adapting both the spoken and unspoken messages between people in order to create and change the perceptions that exist in our minds". It is an important aspect of human communication due to the fact that human beings will always need to communicate and socialize for a peaceful co-existence.

Güngör (2011) further explains in his study that for an interpersonal communication to occur, there must be a minimum number of two and a maximum number of five people as this is a criterion. Also, if the number of people involved in communication exceeds five, then this form of communication would be described as group communication. Thus, for in interpersonal communication the number of people serves an indicator (Güngör, 2011; Seviik, 2013). Sevik (2013) found that interpersonal communication can be divided into three different types which includes verbal, non-verbal and written communication. Interpersonal communication helps people realize themselves and become friends with other people.

The role of interpersonal communication in human communication cannot be over-emphasised as this has been identified to be an influential factor in any meaningful human encounter. This is in the sense that humans generally relate better with each other and tend to achieve more when there is a meaningful exchange of ideas at the interpersonal level. As a matter of fact, other forms of human communication such as group and mass communication, sometimes begin with the interpersonal aspect which are later expanded to other context.

\section{Face-to-Face Interaction and social media usage among youths}

The notion of face-to-face interaction has drawn the attention of many scholars right from the earliest $20^{\text {th }}$ century (Nardi \& Whittaker, 2002). This form of interpersonal communication involves the use of verbal and non-verbal messages such as facial expressions, body movements and vocal cues between two or more people. It also pervades all other forms of communication because it is mostly used by a lot of people. Sociologist Georg Simmel (1908) explained that the sensory organs of the body perform a crucial role in interaction while also discoursing instances of human behaviour such as an eye contact. Face-to-face interaction occurs when people who are in the same physical space exchange information, thoughts and feelings using eye contacts, facial expressions, body movements, or verbal messages.

According to Acacio (2012), several components that differentiates the face-to-face communications from 
other forms of communication include the fact that it facilitates the effectiveness of meaning because it allows individuals reach a conclusion faster when there is an issue. For example, a five minutes face-to face conversation would supersede a 15 minutes e-mailing or texting and waiting for replies. Non-verbal communication is another factor that differentiates face-to-face communication from other forms of communication. This involves understanding, reacting and adapting to non-verbal messages such as symbols, body movements and posture. For instance, acts such as when a person repeatedly checks the wristwatch or yawns could signify tiredness or time to wrap things up. Face-to-face interaction offers personal touch or personal contact which helps to develop a feeling of warmness and appreciation. This can easily be shown when people are together.

In the past, people solely relied on traditional media or interpersonal communication to share and receive information. The emergence of social media has altered the way people interact and communicate as the social media is becoming increasingly popular and commonly used by people in modern times more than ever. It owes its birth to the creation of Web 2.0 technologies and the versions that followed. According to Kaplain and Haenlein (2010), social media is "a collection of web-based applications that develop on the bases of the ideology and technology of web 2.0, and allows the production and exchange of user generated contents". The design of the previous version, Web 1.0 had constraints that impeded the creation of content useful for these sites but the advent of Web 2.0 brought about limitless opportunities (Krishnamurthy \& Cormode, 2008; Jepngetich, 2015). Gunduz (2017) found that social media comprises of individual platforms that enable every digital/virtual user with cellular network access to share content, express opinions about topics or issues of concern and most importantly, serve as an avenue for communication or interaction processes to take place without any time or place constraints. The first social media platform known as SixDegree.com was introduced in 1997. It provided users the ability to create and personalize their profile pages, upload pictures and interact. In 2002, more social networking sites like Friendster, and Myspace began to surface.

Basically, each of the social media platforms serve different uses. Kaplain \& Hanlein. (2010) categorised social media into six groups such as "collaborative projects (for example, Wikipedia), Blogs and Micro Blogs (for example, Twitter), Content Communities (for example, You-tube and Daily Motions), Social Networking Sites (for example, Facebook), Virtual game worlds (for example, World of War craft), and Virtual Social World (e.g. Second life)". Social media can be employed for both serious and trivial issues. For example, people can use social media to watch video as well as engage in business activities. Social media can be used to organize campaigns whether political or non-political, create awareness, engage in social interaction, marketing, and healthcare activities. Oyesomi, and Okorie (2014) describe social media as an innovative pattern of building friendships with different sites introducing opportunities for people to improve their social networks.

According to Lewis (2009) cited in Sponcil and Gitimu (2013), "students in higher institutions are the main consumers of social media". It is becoming popular and widely used amid criticisms as a result of the fact that it has changed the way people communicate, interact, investigate, and socialise (Aisar, Mohd \& Nur, 2015). The teenagers and youths grew up getting familiarized with various types of social media. Kaplan and Hanlein (2010) observes that they are easily becoming digital literate and succeeding in the digital environment where adults are just naturalized people. The ability to interact and stay connected with their loved ones influences people to use social media.

In spite of this trend, researchers have observed that face-to-face communication remains an essential part of the social system (Christopher, 2008; Stenberg, 2012). Regardless of the introduction of numerous innovative information and communication technologies, face-to-face communication remains the most commonly used and general form of communication among people. Several theorists recommend face-to-face communication because it engages more human senses than computer mediated communication where communication occurs over one's computer or phone screen (Nardi \& Whittaker, 2002). Emmit and Gorse (2006) also affirmed in their study that face-to- face interaction is the preferred means particularly for resolving problems and contentious issues.

Aisar, Mohd and Nur (2015) examined awareness and usage of social media among mass communication students of Kano State Polytechnic using a mixed method approach. They found a high level of awareness (98.2\%) ease of access to the internet and regular use of mobile phones for social networking while a few (37.8\%) report and share news or information on social media. The qualitative findings reveal that social networks are used for academic purpose, self-expression, and establishing global friendship.

Kassim, Mohd and Baharuddin (2015), found that students have positive perceptions of the use of social media with a mean score of 4.01. The respondents also agreed that social media increases the connectivity of friends and encourages information sharing among friends.

Jepngetich (2016) studied the use and influence of social media on face-to-face communication on the educational development of college students in Eldoret town using the mixed method approach. The study established that social networking sites are quite beneficial to academic development of college students while noting that social media does not to a great extent threaten face to face communication. The study also indicated 
that social media was the preferred form of communication among students in Eldoret town due to its many benefits.

Gapsiso and Wilson (2014) affirmed that the creation of the Internet has shifted communication from interpersonal to computer-mediated among teenagers with their family and friends, it has also strained the ties that bind them by reducing the time spent engaging in face-to-face communication.

Zeital-Bank and Tat (2014) in a study on social media and its effects on individuals and social systems by illustrating the current state of social media usage and the new forms of virtual interaction. Investigating the question on how the brain deals with the modern forms of information technologies and the outcomes for our social behaviour, the study concludes that social media is a powerful source for communication and learning with huge impact on the brain and human lives. The study further revealed that since emotions are important in human communication, social media interaction presents a serious threats for individuals and society.

Investigating social media use and real-life social relationships as well as the social and psychological implications among Nnamdi Azikiwe University, Awka undergraduates in a qualitative study, Godson and Chukwuemeka (2014) found that there are negative effects associated with social media use as a means of social interaction and relationships than with real-life social interaction and relationships. Although the study recorded no clear stance among respondents on which type of communication is suitable for social interactions, majority of the students agreed that they like social media and it has improved their relationships because it allows them to interact easily with their old friends and acquaintances as well as easily build new relationships.

Osazee-Odia (2017) investigated the influence of social media on sociability among university students in Nigeria. The findings disclosed that the age, year of study, family income determines the respondents' ability to explore the benefits of social media utilization for sociability. Also, majority of the respondents noted Facebook as a main platform for friendship sociability, with mobile media as a medium of constructive sociability behaviour and respondents' friendship. The study also revealed that the respondents' frequency of interaction with friendship groups cut across different time frame with communication relations and socio-pleasure as a means of strategic sociability fulfilment.

Nelson (2012) interrogated the effects of technology on interpersonal relationships among Rowan University students' between the ages 18 to 25 years. The study was aimed at determining how young adults are communicating with each other and to understand what is driving them to communicate using different methods of communication. The researcher concluded that what communicators are trying to disseminate determines how they communicate the message just as the urgency of a message influences the choice of the medium. Respondents affirmed that different mediums affect the clarity and persuasiveness of the message just as relationships are affected by how communication occurs. However, majority of the students felt that technology has both positive and negative effects while some felt it connects people more but provides communicators with a place to hide.

While examining social Interactions across media, Lin (2004), compared college students' interpersonal interaction online, face-to-face, and on the telephone using a communication diary to measure the relative amount of social interactions college students conducted online in contrast to face-to-face conversation and telephone calls. The findings revealed that face-to-face communication remained the leading mode of interaction. Participants indicated using the internet as often as using the telephone.

\section{Methodology}

The study adopted the mixed methods research design combining both quantitative and qualitative research approaches in collecting data from a population of 39,214 (total number of students enrolled in the University of Lagos for the 2018/2019 academic session). A sample size of 396 was determined using a sample size calculation formula. Data was collected with the use of questionnaire and a focus group guide. Final respondents were drawn through a multistage sampling technique which began with the selection of two faculties (Education and Social Sciences) out of 12 systematically. At the next stage, social sciences was purposively picked due to the fact that students in this faculty tend to be more active and more knowledgeable users of the social media given the nature of their field of study. Two departments were systematically selected from the Social Sciences faculty to complete the process. The discussions from the focus group was audio-taped with the consent of the participants and transcribed thematically.

\section{Findings and Discussions}

\section{The pattern of social media usage among Unilag undergraduates}

The findings indicated that majority of the students $(95.6 \%)$ possess internet accessible devices. Some of the students indicated that they make use of social media platforms like Facebook, WhatsApp, Tinder, WordPress, Twitter among others. Facebook ranked as the most commonly used (31\%) social media platform. Majority of the respondents also spend 6 to 9 hours daily on social media regularly. Recent studies have discovered that the quantity of time people spend on the internet especially on social media is ever increasing (Tang \& Cheah, 2017). 
This suggests a level of dependence as the media dependency theory posits that a prolonged use of a medium leads to a close relation of dependence in the audience implying that people are becoming more dependent as they see it as a means to attain their goals. Since the students spend much time on social media, a level of dependence is established.

The findings of the study in relation to pattern of social media usage affirms the findings of Chukwuere and Bonga (2018) who noted that many students utilised social media and social networking sites excessively. Also, majority of the participants in the focus group discussion attested that they expend much time on social media. The findings verified the constant presence of students on social media platforms noted in the study of Aisar, Mohd and Nur (2015) who observed that majority of the students are knowledgeable of social networks and access the internet regularly through the use of their mobile phones.

Also, majority of the students affirmed that they use social media for communication $(80.3 \%)$, information (76.9\%), and entertainment (71.5\%) purposes. Thus, this correlates with the findings of Aisar et al. (2015) as they noted that students also utilize social networks for academic purpose, self-expression, and establishing a global friendship. Researchers have observed that social media has enabled the spread and distribution of content messages that draw the attention of students or teenagers (Liangfei, Qian \& Whinston, 2015).Thus, in as much as social media has improved communication, it has also changed other aspect of life such as entertainment. The Uses and Gratification theory posit that people actively make use of certain media that best satisfies their needs therefore, majority of the students may have picked Facebook as the mostly used platform because it provides an avenue for communication, information and entertainment purposes. Similarly, the study of Aisar et al (2015) indicated that the uses and gratification theory is practical as well as significant to the study of social media as majority of the students employ social networking sites to make friends from far places, report and share information, conduct researches and socialize themselves.

\section{The use of face-to-face interaction among students}

Concerning the use of face-to-face interaction, majority of the students $(64.1 \%)$ agreed that it is for important discussion and that it enables them relate better with their friends and colleagues unlike social media interactions. Similarly, majority of the respondents (75.7\%) agreed that face-to-face interactions passes across their message better than social media interactions and also $(75.6 \%)$ of the respondents were of the opinion that face-to-face interactions is more spontaneous than social media interactions. Some of the participants in the focus group discussion added "interaction through face-to-face allows me to read emotions of people during conversations because on social media, people tend to lie; also people freely express themselves face-to-face without getting misunderstood". According to findings of Nelson (2012), emotions associated to a message is significant in how a person chooses to communicate a message. For instance, in a situation whereby a person has something emotional to say, he/she may prefer communicating by a phone call or communicate face to face. The findings of the study also revealed that different mediums of communication influence the clarity and persuasiveness of a message. The findings is also in line with Zeital-Bank and Tat (2014) research results which indicated that emotions perform an important function in human communication.

Furthermore, the findings of Jijian (2016) reveals students prefer to face-to-face interaction over talking online regardless of how much time expended on social media. Therefore, the nature of face-to-face interaction determines how it is used. This is in conformity with media richness theory which explains that people make use of face-to-face interaction because of its richness nature and its perceived helpfulness in achieving their communication needs. Hence, face-to-face communication remains a necessary form of communication

\section{The perceived difference between online and face-to-face interactions by Unilag Undergraduates}

This research question is correlation with the Media Richness theory which posits that people make use of certain mediums because of the richness in achieving their needs. The study found out that social media interaction increases personal communication and interaction more than face-to-face interaction as agreed by $72.8 \%$ of the respondents but $65.9 \%$ also agreed that face-to-face conversations are more productive than social media conversations. This implies that face-to-face interaction is still relevant and according to findings of Jepngetich (2016), social media does not to a great extent threaten face to face interaction. Furthermore, face-toface interactions offers a sense of warmth and humanness unlike social media interactions as this was agreed by majority of the respondents $(74.1 \%)$. In a similar way majority of the respondents $(53.6 \%)$ agreed that face-toface interactions builds stronger relationships among people more than social media interactions. According to a participant in the focus group discussion, "there are more things to say to people when communicating face-toface unlike social media where you can easily get bored or run of out of words to say". She also added that she feels accomplished talking to people face-to-face. However, another participant disagreed with this opinion saying, "When I'm talking to people over the phone especially, I'll be able to say anything I want to say without being shy but I can't do this face-to-face". Although, other participants could not really indicate whether interacting on social media is better than interacting face-to-face or whether social media is replacing face-to- 
face interactions. However, majority of the students were of the opinion that each form of interactions have its benefits likewise its drawbacks. The perceived difference between social media interactions and face-to-face interactions explains how people determine what medium of communication is meant to be used for certain situations based on their beliefs. In the findings of Nelson (2012), what people intend to communicate determines how they pass across the message. The importance and urgency of the message can also affect their choice of medium.

\section{The factors influencing social media interactions and face-to-face interactions among Unilag undergraduates}

The study indicated that majority of the participants (73.6\%) asserted that it is faster and easier to communicate on social media than face-to-face interaction. Likewise, $79.8 \%$ agreed that communicating on social media is more convenient than face-to-face interaction. Similarly, majority of the students $(61.8 \%)$ agreed that social media interaction eliminates geographical boundaries and time factor and also social media interaction reduces the stress of traveling distances to communicate. Majority of the respondents $(84.1 \%)$ agreed that they are more confident communicating on social media than face to face interaction and also majority agreed that they are very knowledgeable about how to communicate on social media. Also, majority of the respondents $(81 \%)$ agreed that they would rather discuss important issues face-to-face than on social media. Finally, majority of the respondents (83.1) agreed that they sometimes feel misunderstood when communicating on social media. The participants in focus group discussion added that "peer pressure influences them to make use of social because their friends are on social media so they want to belong and distance is also a factor too because you can interact with your family friends wherever they are. Also, it is faster and easier especially when you want to tell someone something you don't want others to hear". Other participants were of the opinion that "it is faster to communicate face-to-face because you eventually get a reply but on social media, you will have to wait till the person responds". The findings of Zeital-Bank and Tat (2014) research revealed that some of the respondents felt even though social media brings people closer more, it also provides a place for people to hide. People are influenced to make use of certain mediums to achieve their communication needs quickly. According to the findings of Godson and Chukwuemeka (2014), there was no conclusion on which of the two types of communication is more suitable for social relationships. However, majority agreed that they like social media and its use has enhanced their social relationships because they can interact easily with their old friends and acquaintances regardless of distance as well as build new relationships.

\section{Conclusion}

Social media is commonly used by present day youths especially university students. The idea of the world being a global village has been translated into reality through these social media connecting people around the world. It makes people more informed, enlightened, and kept up-to-date with world events. The introduction of technology brought about an improvement in spheres of human life. Information are relayed faster and conveniently but conversations done over social media is not quite the same as those done face-to-face especially depending on the weight of the matters. The use of social media cannot replace face-to-face interaction among people irrespective of how much time they spend on the sites. Regardless, it provides an avenue for youths to communicate with their peers, seek information, and entertain themselves. From the study findings, the respondents employ both mediums of communication because they each have their unique distinct functions.

\section{Recommendations}

Based on the findings of the study, the following recommendations are made:

1. Since students expend much time on social media platforms particularly Facebook for interaction and entertainment purposes instead of focusing on their academic activities or acquiring new skills, there is a need for the university administrators and lecturers to sensitize and educate all students how to make better use of these platforms in a more educative, profitable and productive way so as to improve their lives especially their academic performance.

2. The university should also take a step in enlightening students about the advantages and disadvantages of social media for example, cyberbullying by organizing seminars for them at least once in a month or they can have it as a club for creating awareness on proper usage of social media in building themselves and their future careers. This could be part of their school activities.

3. Parents, lecturers and school administrators should try to familiarize themselves with modern social media platforms because it helps them in understanding how to connect and reach with their children and students.

4. Students are to be encouraged to openly express themselves in their homes and schools as this reinforces the importance of face-to-face interaction.

5. Mass communication department should create a course on social media that teaches youths how to 
benefit from the creative aspect of social media as well as how it can be of benefit to the society.

6. Researchers should carry out further research on this study as there are limited studies concerning this research topic.

\section{References}

Acacio, V. (2012). Benefits of face-to-face communication. Retrieved from http://www.blue jeans.com/Blog/benefits-face-to-face

Aisar, M., Mohd, N. \& Nur, S. (2015). Awareness and Usage of Social Media: A study of Mass communication Students of Kano State Polytechnic. International Conference on Languages. (1-15)

Ball-Rokeach, S. J., \& De Fleur, M. (1976). A dependency model of mass media effects. Communication Research, 3(1), (3-21).

Blumler, J. G., \& Katz, E. (1974). The Uses of Mass Communications: Current Perspectives on Gratifications Research. Beverly Hills, California: Sage Publications.

Boyd, D. (2007, December). Why Youth (Heart) Social Network Sites: The Role of Networked Publics in Teenage Social Life. (David Buckingham ed.) Youth, Identity and Digital Media. Cambridge, MA: MIT Press doi:10.1162/dmal.97802622524834.119

Chukwuere, J. \& Bonga, S. (2018). An exploration in the influence of social media on university students' relationships.

Daft, R. L., \& Lengel, R. H. 1984. Information richness: A new approach to managerial information processing and organizational design. Research in Organizational Behavior 6, 191-233.

Donne, J. (1624). No Man is an Island. Retrieved from https://www.phrases.org.uk/meanings/no-man-is-anisland.html

Godson, O. O., \& Chukwuemeka, O. A. (2014). Social Media Use and Real-Life Social Relationships. Retrieved from https://www.ajol.info/index.php/cajtms/article/view/110621

Jepngetich, K. (2016). Influence of Social Media on Face To Face Communication among College Students: A Study of Selected Colleges in Eldoret.

Kaplan, A. M., \& Haenlein, M. (2010). Users of the world, unite! The challenges and opportunities of social media. Business Horizons, 53-61.

Nardi, B. A. \& Whittaker, S. (2002). The Place of Face-to-Face Communication in Distributed Works. University of Toronto Press.

Osatuyi, B. (2013). Information Sharing on Social Media Sites. Computers in Human Behavior, 29(6), 26222631. doi:10.1016/j.chb.2013.07.001

Seviik, T. (2013). The Influence of Facebook on Interpersonal Communication

Sponcil, M., \& Gitimu, P. (2013). Use of social media by college students: Relationship to communication and self-concept. Journal of Technology Research(4), 1-14

Spring, R. E. (2002). Retrieved September 18, 2018, from http://zimmer.csufresno.edu/ johnca/spch100/7-4uses.html

Tang. S. \& Cheah, S. (2017). Redesigning Learning for Greater Social Impact.71-88. doi: 10.1007/978-981-104223-2

Wei, R., \& Lo, V. (2006). Staying connected while on the move: Cell phone use and social connectedness. New Media Society, 8, 53-72.

Whittaker, S. \& Nardi, B. (2002). The place of face-to-face communication in global context. University of Toronto Press.369.

Yang, L. (n.d.). Encyclopaedia Britannica Incorporation. Retrieved February 5, 2019, from https://www.britannica.com/topic/public-opinion/the-mass-media

Zuo, X. A. (2015, May). The Impacts of Personality, Face-to-Face Interaction, Social Media and Mass Media Use on Perceived Social Support and Intercultural Adaptation Among Mainland Chinese Students in Hong Kong. 